\title{
On sample size calculation in testing treatment efficacy in clinical trials
}

\section{Rownak Jahan Tamanna ${ }^{1}$, M. Iftakhar Alam ${ }^{1}$, Ahmed Hossain ${ }^{2}$, Md Hasinur Rahaman Khan ${ }^{1}$}

\footnotetext{
${ }^{1}$ Institute of Statistical Research and Training, University of Dhaka, Bangladesh, e-mail: rjahan@isrt.ac.bd, e-mail: iftakhar@isrt.ac.bd, e-mail: hasinur@isrt.ac.bd

${ }^{2}$ Department of Public Health, North South University, Bangladesh, e-mail: ahmed.hossain@northsouth.edu
}

\section{SumMary}

Sample size calculation is an integral part of any clinical trial design, and determining the optimal sample size for a study ensures adequate power to detect statistical significance. It is a critical step in designing a planned research protocol, since using too many participants in a study is expensive, exposing more subjects to the procedure. If a study is underpowered, it will be statistically inconclusive and may cause the whole protocol to fail. Amidst the attempt to maximize power and the underlying effort to minimize the budget, the optimization of both has become a significant issue in the determination of sample size for clinical trials in recent decades. Although it is hard to generalize a single method for sample size calculation, this study is an attempt to offer something that might be a basis for finding a permanent answer to the contradictions of sample size determination, by the use of simulation studies under simple random and cluster sampling schemes, with different sizes of power and type I error. The effective sample size is much higher when the design effect of the sampling method is smaller, particularly less than 1. Sample size increases for cluster sampling when the number of clusters increases.

Key words: clinical trial, power, sample size, type I error

\section{Introduction}

In statistics, quality assurance, and survey methodology, sampling is concerned with selecting a subset of individuals from a population to estimate the characteristics of the whole population. Each observation measures one or more properties (such as weight, location, color) of observable bodies 
distinguished as independent objects or individuals. The aforementioned subset of observations is known as a sample. It is considered to be representative of the population. It must be selected in such a way that any conclusions drawn from studying the sample can be generalized.

Logically, the first major decision in choosing a good sample is the choice of the sample size. At the initial stage of study design, an important consideration is to determine exactly how many observations to include in the study. Sample size determination is probably the most important component of planning a statistical study. An adequate size is required to obtain a reliable result.

If the sample size is too small, important treatment differences can easily go undetected. However, this does not mean that an investigator should enroll as many patients or animals as possible in the study. If the number of patients or animals exceeds the number required, the study will be unnecessarily expensive, prolonged, and sometimes unethical. An investigator will have to balance enrolling sufficient patients or animals to detect relevant treatment effects against ensuring that important resources are not wasted.

The concerns and challenges of sample size determination may vary depending on the purpose of the study. But the aforementioned risk means that the determination of sample size is a crucial specification in clinical trials. A clinical trial is any research study that prospectively assigns human participants or groups of humans to one or more health-related interventions to evaluate the effects on health outcomes. Clinical trials usually involve two groups of individuals - a case group to be given the health-related intervention and a placebo group to be given an inactive treatment, to determine whether or not the intervention offers the desired effect (Carlin et al., 2010). There are different ideas on how sample size should be calculated and interpreted. Prashant shows through a simple example how one can calculate required sample size (Kadam and Bhalerao, 2010). However, in general, the larger the number of participants in the trial, the greater the opportunity to identify a significantly different treatment effect. Use of a larger number of people ensures that any observed difference is due to the analyzed effect, rather than random fluctuations (Wittes, 2002).

The availability of individuals in clinical trials is restricted by many factors. There is also a financial concern to balance the desire to give a trial a high probability of identifying a treatment effect with the increasing cost of recruiting more test subjects. Suppose a new treatment is for a condition that already has a drug that substantially improves the quality of life of 
sufferers. In that case, it might be ethically unsound to assign more patients than necessary to the new alternative, as the trial participants may receive inferior treatment. The trial's sample size must balance the clinical, financial, and ethical needs of the sponsor, trial participants, and potential future treatment recipients. Adequate sample size has a great impact on a clinical trial. By improving power analysis, the sample size can be made more accurate (Castellini et al., 2016). Despite improvements, the reporting of sample size calculation and power analysis remains inadequate and often erroneous (Charles et al., 2009). This paper covers the essentials of calculating power and sample size for a variety of sampling designs.

The paper is organized as follows. Section 2 contains more discussion of sample sizes in clinical trials. The methodology is described in section 3 . Section 4 presents the numerical results obtained from a simulation study. Finally, conclusions are given in section 5 .

\section{Sample size in clinical trials}

Conducting a clinical trial requires much preparation, and an essential aspect of designing a clinical trial is determining the correct sample size for that trial. Over the last decade, there has been considerable interest in sample size estimation in the design of clinical trials. Determining the correct sample size saves money and resources. There are many ways in which sample size can be calculated, and all of these methods aim to find the best sample size or the smallest sample size necessary to get accurate and inference-worthy results (Sakpal, 2010). Power analysis has a major impact on sample size calculation in clinical studies (Suresh and Chandrashekara, 2015; Joones et al., 2003).

Phase II clinical trials are generally single-arm studies aimed at estimating the activity of a new treatment. Phase II trials decide whether the new treatment is promising and warrants further investigation in a largescale randomized phase III clinical trial, based on an observed response rate that appears to improve on the standard treatment or other experimental treatments. Since the sample size is small (generally less than 50 patients), phase II trials are only able to detect a large treatment improvement (Khan et al., 2012). Fleming's single-stage procedure (Fleming, 1982; Machin et al., 1997b) has been a widely used approach in early-phase drug development.

The sample size method in a single-stage Fleming's design uses a nor- 
mal approximation to the binomial distribution. The sample sizes obtained using this approximation result in differences in sample sizes compared with exact methods, particularly for relatively small studies. This is discussed by A'hern (2001), who also provided sample size tables using exact methods, which are larger than those obtained using the design proposed in by Fleming (1982). A'hern (2001) provides sample sizes based on approximate levels and power. Sample size software (Machin et al., 2011) and tables (A'hern, 2001; Machin et al., 1997) are available for the Fleming design and A'hern exact sample sizes.

A sample size formula for the stratified log-rank test is discussed in Ahnn and Anderson (1998). They also describe how one can utilize these generalized formulas in calculating sample sizes and assessing power in complex multi-arm clinical trials. Moussa (1988) discusses the Bernstein and Lagakos design, which is suited for exponential survival due to its generality and allowance for stratification. In contrast, the Freedman design is convenient for distribution-free survival because of its simplicity. With other fixed specifications, the saving on sample size becomes minimal as the accrual period or post-accrual follow-up time units increase above three. Required sample size also increases drastically when the number of strata exceeds three.

Phase III studies are extensive randomized comparative experiments typically with time-to-event outcomes (e.g., overall survival) (Sargent and Taylor, 2009). These trials are designed to provide definitive information to guide clinical practice. Wang et al. (2006) investigate the impact of using various phase II effect size estimates on sample size planning for phase III trials. In general, if the point estimate of the phase II trial is used for planning, it is advisable to size the phase III trial by choosing a smaller alpha level or a higher power level.

There are also some ethical considerations in the choice of sample sizes in clinical trials. An undersized study can expose subjects to unnecessary (sometimes potentially harmful or futile) treatments without the capability to advance knowledge. An oversized study has the potential to expose an unnecessarily large number of subjects to possibly harmful or futile treatments (Altman, 1980). Corrected sample size calculation is a major issue in the conduct of clinical trials. Bethan shows that sample size calculations in trials of hip and knee osteoarthritis are not adequately reported (Copsey et al., 2018). However, over time, improvements are being made in relation to the problem of inadequate sample size. For example, sample size calculation in endodontic clinical trials improved significantly in 2010 compared 
with 2000 (Shahravan et al., 2014). Sample size calculation is an essential part of study design, serving to ensure the validity, accuracy, reliability, and scientific and ethical integrity of the study.

\section{Method}

In different clinical trials, the sampling procedures are also different. Randomization as a method of experimental control has been extensively used in clinical trials and other biological experiments. It prevents selection bias and insures against accidental bias. Randomization based on a single sequence of random assignments is known as simple randomization. This technique maintains the complete randomness of the assignment of a subject to a particular group. This randomization approach is simple and easy to implement in clinical research. In extensive clinical research, simple randomization can be trusted to generate similar numbers of subjects among the groups. However, randomization results may be problematic in clinical research with relatively small sample size, resulting in unequal numbers of participants among the groups.

Cluster randomized trials, where individuals are randomized in groups, are increasingly being used in health-care evaluation. In many situations, the use of a cluster-randomized trial may be advantageous. For example, when evaluating interventions targeted at the health professional (such as the impact of educational training on good clinical practice), randomizing at the level of the professional rather then the individual patient may be the only feasible method of conducting a randomized trial. Similarly, when assessing a dietary intervention, it is common to randomize families as an intact unit to avoid the possibility of different members of the same family being assigned to different interventions.

\subsection{Comparison of means}

We compare the two treatments in a clinical trial with population means $\mu_{1}$ and $\mu_{2}$ of the measurements of interest. We also assume that the populations have equal variance $\sigma^{2}$, which is unknown to the analysts. Let the treatment measurements for each population follow separate normal distributions with $\mu_{1}$ and $\mu_{2}$ as the parameters. The performance of two treatments can be tested with the use of a two-sample $t$-test procedure, since we can estimate the variance parameter using a sample. The hypothesis can then be set up 
as

$$
H_{0}: \mu_{1}-\mu_{2}=\mu_{d}=0
$$

versus

$$
H_{1}: \mu_{1}-\mu_{2}=\mu_{d} \neq 0 .
$$

Under the assumption of large sample theory with finite population variance, the underlying $Z$ pivotal is approximated as a normal distribution. The sample size is then calculated using the following formula:

$$
n=\frac{\left(Z_{\alpha / 2}+Z_{\beta}\right)^{2}\left(2 \sigma^{2}\right)}{d^{2}},
$$

where $\alpha$ is the probability of type I error, $\beta$ is the probability of type II error, $d$ is the clinically meaningful difference, and $n$ is the required sample size for each group.

\subsection{Comparison of proportions}

Now we compare two treatment groups in a clinical trial with population proportions $p_{1}$ and $p_{2}$. Assume that the first population represents the subjects under the standard treatment and the second population represents the subjects under the new treatment. Then the numbers of subjects who benefited from the treatments are separately distributed binomially, with the proportions as the parameters. The performance of the two treatments can be tested with the two-sample $Z$-test procedure. The hypothesis can then be set up as

$$
H_{0}: p_{1}-p_{2}=p_{d}=0
$$

versus

$$
H_{1}: p_{1}-p_{2}=p_{d} \neq 0 .
$$

Under the assumption of large sample theory with finite population variance, the underlying $Z$ pivotal is approximated as a normal distribution. The sample size is then calculated using the following formula:

$$
n=\frac{\left(Z_{\alpha / 2}+Z_{\beta}\right)^{2}\left[p_{1}\left(1-p_{1}\right)+p_{2}\left(1-p_{2}\right)\right]}{d^{2}},
$$

where $d$ represents the proportional difference $p_{1}-p_{2}$. 


\subsection{Adjustment for cluster design}

Adjustment of the sample size for cluster design is needed to obtain an effective sample size for a study. To calculate the appropriate sample size adjustment for clustering, the design effect inflates standard sample size estimates for individually randomized designs. The effective sample size $(E S S)$ is

$$
E S S=\frac{m k}{D E}
$$

where $m k=n$ and $D E=1+(1-m) \rho$ (Campbell et al., 2004). Note that $D E$ is the design effect, $m k$ is the total number of subjects in all clusters, $m$ is the number of subjects in a cluster, $k$ is the number of clusters, and $\rho$ is the intra-cluster correlation coefficient.

\section{Numerical studies}

We calculate sample size for different values of type I error, power, mean and proportional difference, design effect, and number of clusters. Table 1 represents the sample sizes that are obtained when the fixed mean difference is assumed to be 7 units.

Table 1. Estimated sample size for fixed mean difference $(d=7)$

\begin{tabular}{|c|c|c|c|c|c|c|c|}
\hline Type I error & Power (\%) & Sample size & $\begin{array}{c}\text { Sample size } \\
\text { (cluster design) } \\
\mathrm{DE}=0.4\end{array}$ & $\begin{array}{c}\text { Sample size } \\
\text { (cluster design) } \\
\mathrm{DE}=0.5\end{array}$ & $\begin{array}{c}\text { Sample size } \\
\text { (cluster design) } \\
\mathrm{DE}=0.6\end{array}$ & $\begin{array}{c}\text { Sample size } \\
\text { (cluster design) } \\
k=10\end{array}$ & $\begin{array}{c}\text { Sample size } \\
\text { (cluster design) } \\
k=5\end{array}$ \\
\hline \multirow{3}{*}{.01} & 95 & 164 & 410 & 328 & 273 & 94 & 63 \\
\hline & 85 & 120 & 300 & 240 & 200 & 77 & 56 \\
\hline & 75 & 97 & 242 & 194 & 162 & 67 & 51 \\
\hline \multirow{3}{*}{.02} & 95 & 145 & 362 & 290 & 242 & 88 & 60 \\
\hline & 85 & 104 & 260 & 208 & 173 & 72 & 52 \\
\hline & 75 & 83 & 208 & 166 & 138 & 61 & 46 \\
\hline \multirow{3}{*}{.03} & 95 & 134 & 335 & 268 & 223 & 84 & 58 \\
\hline & 85 & 94 & 235 & 188 & 157 & 67 & 49 \\
\hline & 75 & 74 & 185 & 148 & 123 & 57 & 44 \\
\hline \multirow{3}{*}{.04} & 95 & 126 & 315 & 252 & 210 & 79 & 57 \\
\hline & 85 & 88 & 220 & 176 & 147 & 63 & 48 \\
\hline & 75 & 68 & 170 & 136 & 113 & 52 & 41 \\
\hline \multirow{3}{*}{.05} & 95 & 119 & 298 & 238 & 198 & 77 & 55 \\
\hline & 85 & 82 & 205 & 164 & 137 & 61 & 47 \\
\hline & 75 & 64 & 160 & 128 & 107 & 51 & 40 \\
\hline
\end{tabular}

We find from Table 1 that as the power of the test increases, the required sample size for the study also increases. For fixed 0.01 type I error probability, 7 unit mean difference, and $75 \%$ power, the required sample size is 97 . When the power of the study is increased to $85 \%$, the sample size 
also increases to 120 , and when the power of the study becomes $95 \%$, the sample size increases to 164 . We note that this increasing trend in sample size with an increase in the power of the test is the same for any sampling technique with a different design effect.
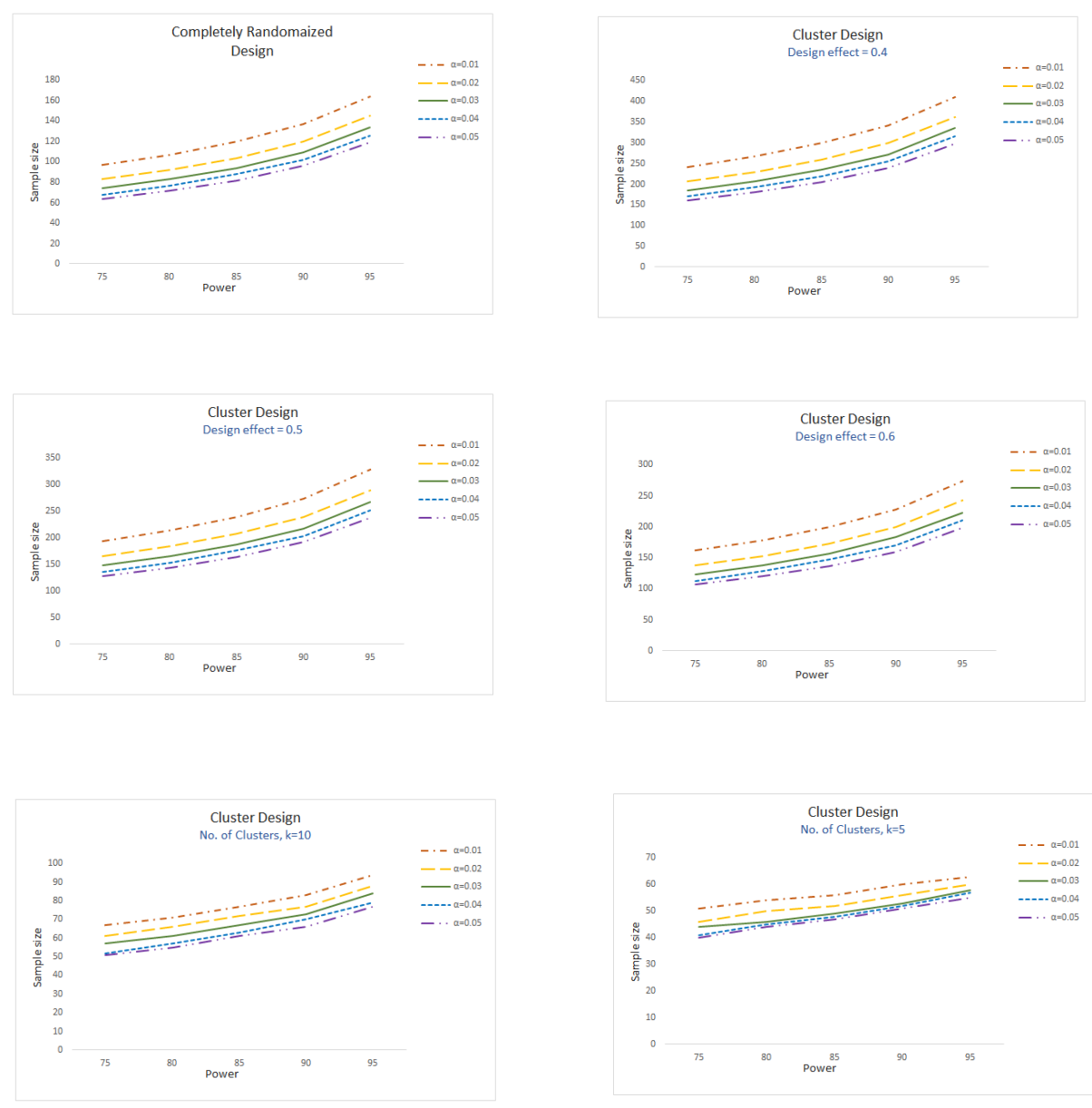

Figure 1. Sample sizes for different values of type I error $(\alpha)$ and power

As seen in Table 1, the required sample size for the study decreases substantially with the increase in type I error. For a fixed power of the test $(95 \%)$, mean difference ( 7 units), and type I error probability (0.01), the required sample size is 164 . When the type I error probability increases to 0.03 , the 
required sample size falls to 134 , and when the type I error probability increases further to 0.05 , the required sample size becomes still smaller (119). This decreasing trend in sample size is due to the increase in type I error. This is the same as we would observe for any sampling technique with a different design effect. A similar trend is obtained for proportional difference, as seen in Table 3.
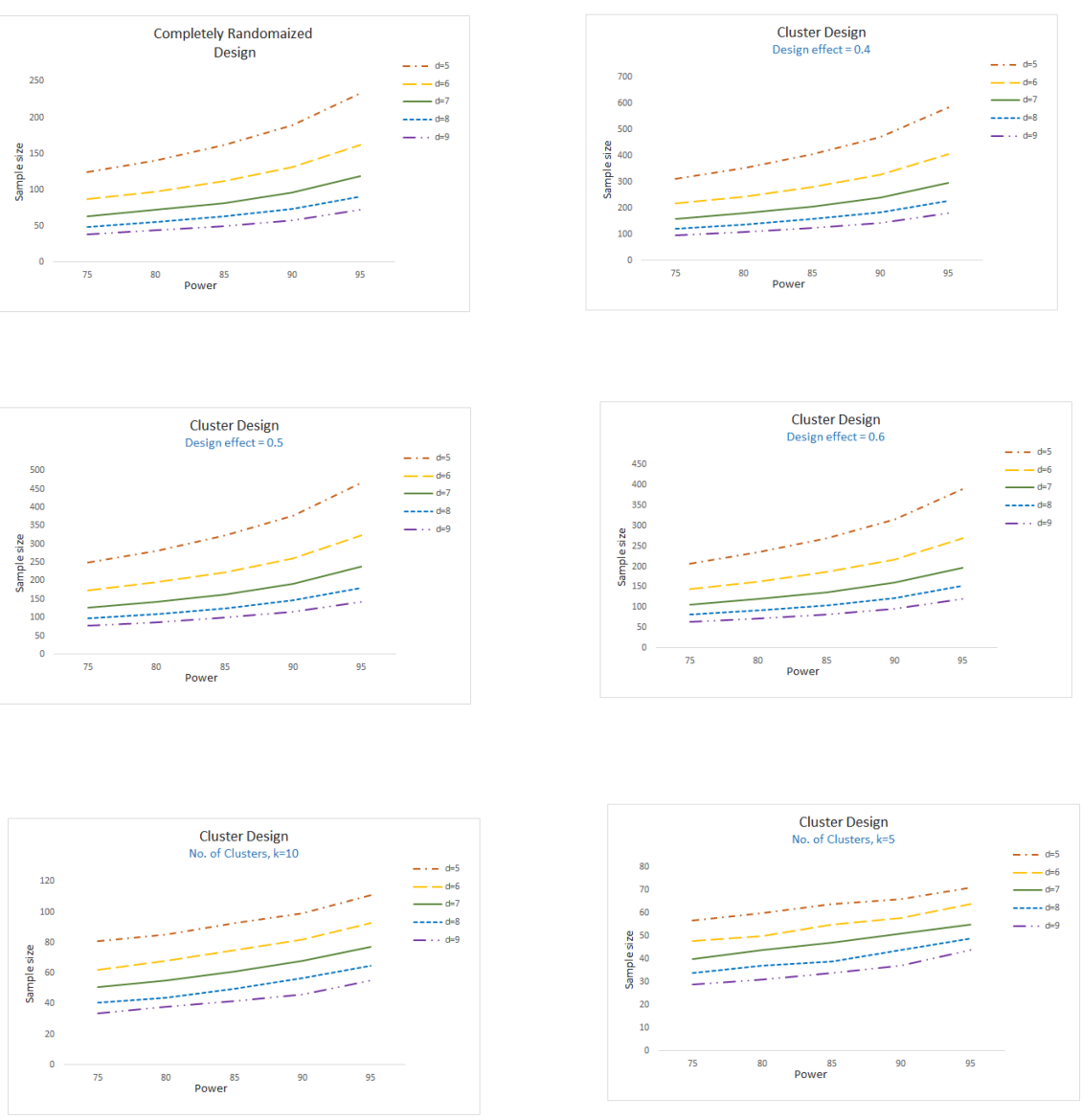

Figure 2. Sample sizes for different values of mean difference and power

Table 2 shows the sample size estimated when type I error probability is assumed to be 0.05 . In treatment comparison, if the mean difference between two treatments increases, the required sample size for the study becomes 
Table 2. Estimated sample size for fixed type I error $(\alpha=0.05)$

\begin{tabular}{cccccccc}
\hline Difference & Power (\%) & Sample size & $\begin{array}{c}\text { Sample size } \\
\text { (cluster design) } \\
\text { DE=0.4 }\end{array}$ & $\begin{array}{c}\text { Sample size } \\
\text { (cluster design) } \\
\text { DE=0.5 }\end{array}$ & $\begin{array}{c}\text { Sample size } \\
\text { (cluster design) } \\
\text { DE=0.6 }\end{array}$ & $\begin{array}{c}\text { Sample size } \\
\text { (cluster design) } \\
k=10\end{array}$ & $\begin{array}{c}\text { Sample size } \\
\text { (cluster design) }\end{array}$ \\
\hline \multirow{2}{*}{5} & 95 & 234 & 585 & 468 & 390 & 5 \\
& 85 & 162 & 405 & 324 & 270 & 93 & 71 \\
& 75 & 125 & 312 & 250 & 208 & 81 & 54 \\
\hline \multirow{2}{*}{6} & 95 & 162 & 405 & 324 & 270 & 93 & 64 \\
& 85 & 112 & 280 & 224 & 187 & 75 & 55 \\
& 75 & 87 & 218 & 174 & 145 & 62 & 48 \\
\hline \multirow{2}{*}{7} & 95 & 119 & 298 & 238 & 198 & 77 & 55 \\
& 85 & 82 & 205 & 164 & 137 & 61 & 47 \\
& 75 & 64 & 160 & 128 & 107 & 51 & 40 \\
\hline \multirow{2}{*}{8} & 95 & 91 & 228 & 182 & 152 & 65 & 49 \\
& 85 & 63 & 158 & 126 & 105 & 50 & 39 \\
\hline \multirow{2}{*}{9} & 75 & 49 & 122 & 98 & 82 & 41 & 34 \\
& 95 & 72 & 180 & 144 & 120 & 55 & 44 \\
& 85 & 50 & 125 & 100 & 83 & 42 & 34 \\
\hline
\end{tabular}

much lower. For 0.05 type I error probability, 95\% test power, and 5 unit mean difference, the required sample size is estimated as 234 . However, when the difference increases to 7 , the required sample size falls to 119 . When the difference further increases to 9, the required sample size becomes still smaller (72). A similar trend is observed for proportional difference, as seen in Table 4. For example, when the fixed type I error probability is 0.05 , the power of the test is $95 \%$, and the proportional difference is set to 0.2 , the required sample size is 149 . When the difference increases to 0.3 , the required sample size is reduced to 59 , and when the difference further increases to 0.4 , the required sample size becomes even smaller (28). Hence, the decreasing trend in sample size due to an increase in the difference (mean or proportion) between the two groups is the same as for any sampling technique with a different design effect.

We have found in treatment comparison that the required sample size is much larger in cluster sampling than in simple random sampling (when the design effect is less than 1). We also notice that as the design effect increases in cluster sampling, effective sample size decreases. As seen in Table 1, for type I error probability 0.05 , test power $95 \%$ and mean difference 7 units, the sample size estimated for simple random sampling is 119 , while for cluster sampling, with design effect 0.4 the sample size is estimated as 298 , and with design effect 0.5 and 0.6 , the sample sizes are estimated as 238 and 198, respectively. This means that the smaller the variance of the sampling technique, the larger the sample size will be.

Table 3 gives the sample sizes that are obtained when the proportional difference is assumed to be fixed at 0.2 units. We found the same trend 

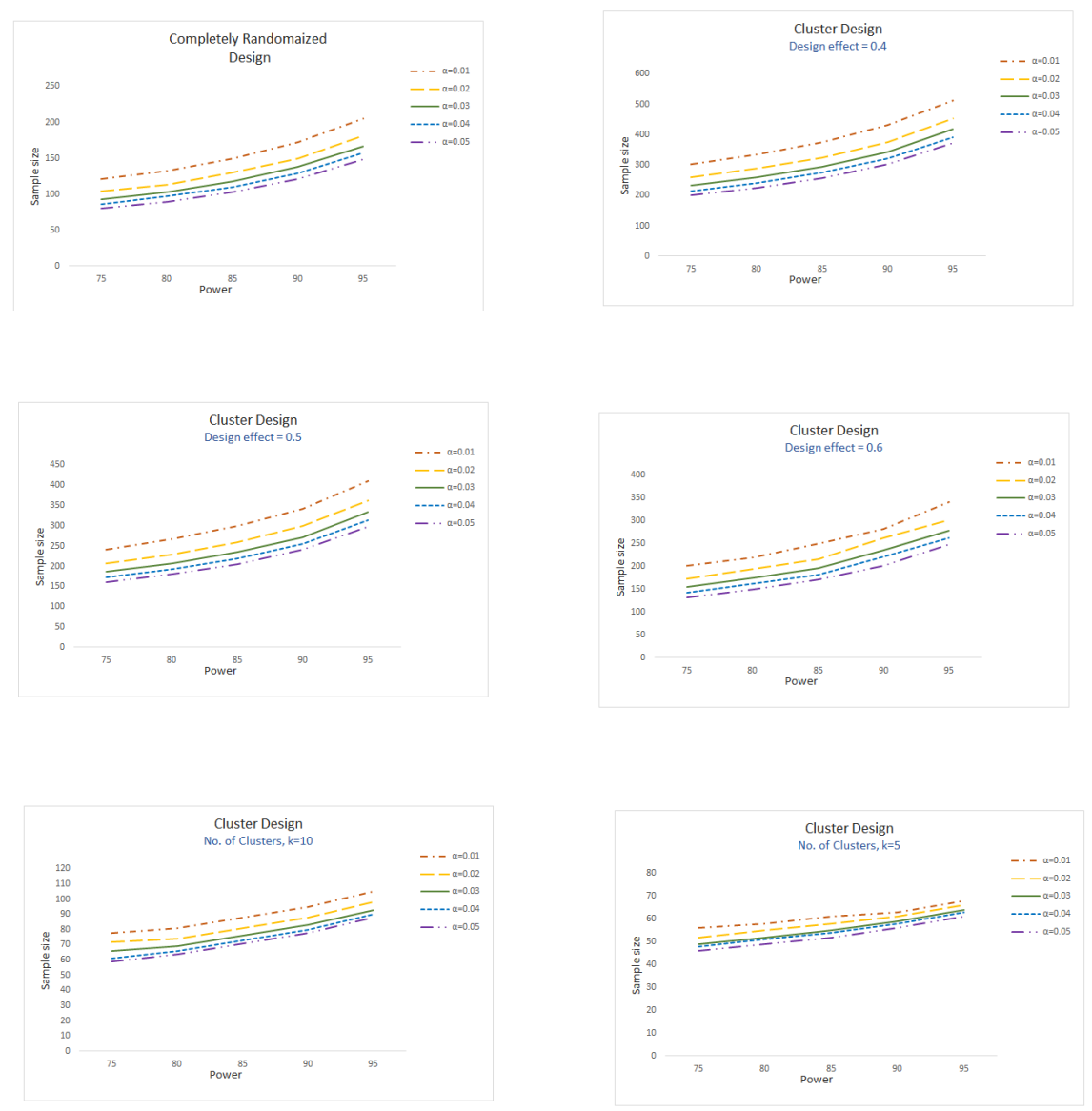

Figure 3. Sample sizes for different values of type-I-error $(\alpha)$ and power

shown in Table 1, in that as the power of the test increases the required sample size for the study increases, and as type I error increases the required sample size decreases.

Table 4 shows the sample size estimated when the proportional difference is assumed to be 0.2 . We note that the required sample size is much lower in cluster sampling than in simple random sampling (when the design effect is greater than 1). It is also revealed that as the number of clusters decreases, the sample size decreases. For example, from Table 4 for type I error probability 0.05 , test power $95 \%$ and proportional difference 0.2 , the sample size required for simple random sampling is found to be 149 . In 
Table 3. Estimated sample size for fixed difference in proportion $(d=0.2)$

\begin{tabular}{|c|c|c|c|c|c|c|c|}
\hline Type I error & Power (\%) & Sample size & $\begin{array}{c}\text { Sample size } \\
\text { (cluster design) } \\
\mathrm{DE}=0.4\end{array}$ & $\begin{array}{c}\text { Sample size } \\
\text { (cluster design) } \\
\mathrm{DE}=0.5\end{array}$ & $\begin{array}{c}\text { Sample size } \\
\text { (cluster design) } \\
\mathrm{DE}=0.6\end{array}$ & $\begin{array}{c}\text { Sample size } \\
\text { (cluster design) } \\
k=10\end{array}$ & $\begin{array}{c}\text { Sample size } \\
\text { (cluster design) } \\
k=5\end{array}$ \\
\hline \multirow{3}{*}{.01} & 95 & 205 & 512 & 410 & 342 & 105 & 68 \\
\hline & 85 & 150 & 375 & 300 & 250 & 88 & 61 \\
\hline & 75 & 121 & 302 & 242 & 202 & 78 & 56 \\
\hline \multirow{3}{*}{.02} & 95 & 181 & 452 & 362 & 302 & 98 & 66 \\
\hline & 85 & 130 & 325 & 260 & 217 & 81 & 58 \\
\hline & 75 & 104 & 260 & 208 & 173 & 72 & 52 \\
\hline \multirow{3}{*}{.03} & 95 & 167 & 418 & 334 & 278 & 93 & 64 \\
\hline & 85 & 118 & 295 & 236 & 197 & 76 & 55 \\
\hline & 75 & 93 & 232 & 186 & 155 & 66 & 49 \\
\hline \multirow{3}{*}{.04} & 95 & 157 & 392 & 314 & 262 & 90 & 63 \\
\hline & 85 & 110 & 275 & 220 & 183 & 73 & 54 \\
\hline & 75 & 86 & 215 & 172 & 143 & 61 & 48 \\
\hline \multirow{3}{*}{.05} & 95 & 149 & 372 & 298 & 248 & 88 & 61 \\
\hline & 85 & 103 & 258 & 200 & 172 & 71 & 52 \\
\hline & 75 & 80 & 200 & 160 & 133 & 59 & 46 \\
\hline
\end{tabular}

Table 4. Estimated sample size for fixed type I error $(\alpha=0.05)$

\begin{tabular}{|c|c|c|c|c|c|c|c|}
\hline $\begin{array}{c}\text { Proportional } \\
\text { difference }\end{array}$ & Power (\%) & Sample size & $\begin{array}{c}\text { Sample size } \\
\text { (cluster design) } \\
\mathrm{DE}=0.4\end{array}$ & $\begin{array}{c}\text { Sample size } \\
\text { (cluster design) } \\
\mathrm{DE}=0.5\end{array}$ & $\begin{array}{c}\text { Sample size } \\
\text { (cluster design) } \\
\mathrm{DE}=0.6\end{array}$ & $\begin{array}{c}\text { Sample size } \\
\text { (cluster design) } \\
k=10\end{array}$ & $\begin{array}{c}\text { Sample size } \\
\text { (cluster design) } \\
k=5\end{array}$ \\
\hline \multirow{3}{*}{.20} & 95 & 149 & 372 & 298 & 248 & 88 & 61 \\
\hline & 85 & 103 & 258 & 206 & 172 & 71 & 52 \\
\hline & 75 & 80 & 200 & 160 & 133 & 59 & 46 \\
\hline \multirow{3}{*}{.25} & 95 & 91 & 228 & 182 & 152 & 65 & 49 \\
\hline & 85 & 63 & 158 & 126 & 105 & 50 & 39 \\
\hline & 75 & 49 & 122 & 98 & 82 & 41 & 34 \\
\hline \multirow{3}{*}{.30} & 95 & 59 & 148 & 118 & 98 & 47 & 38 \\
\hline & 85 & 41 & 102 & 82 & 68 & 36 & 30 \\
\hline & 75 & 32 & 80 & 64 & 53 & 29 & 26 \\
\hline \multirow{3}{*}{.35} & 95 & 40 & 100 & 80 & 67 & 35 & 30 \\
\hline & 85 & 28 & 70 & 56 & 47 & 25 & 22 \\
\hline & 75 & 21 & 52 & 42 & 35 & 20 & 18 \\
\hline \multirow{3}{*}{.40} & 95 & 28 & 70 & 56 & 47 & 25 & 22 \\
\hline & 85 & 19 & 48 & 38 & 32 & 18 & 17 \\
\hline & 75 & 15 & 38 & 30 & 25 & 14 & 12 \\
\hline
\end{tabular}

contrast, for cluster sampling with the number of clusters as 10 , the sample size becomes 88 . When the number of clusters is 5 , the sample size is 61 . Hence, if the number of clusters increases, the sample size increases as well.

\section{Conclusion}

This study reveals that sample size estimation is important in clinical trials, and differs for different sampling methods. The study does not suggest which sampling procedure is better; instead, it gives suggestions about the effective sample size of a study after selecting the sampling technique. This study will be helpful for those who want to work in clinical trials for efficacy testing for two treatments and who do not know much about the required sample 
size a priori. The study shows how sample size varies for different sampling methods and for different levels of test power and type I error rate.
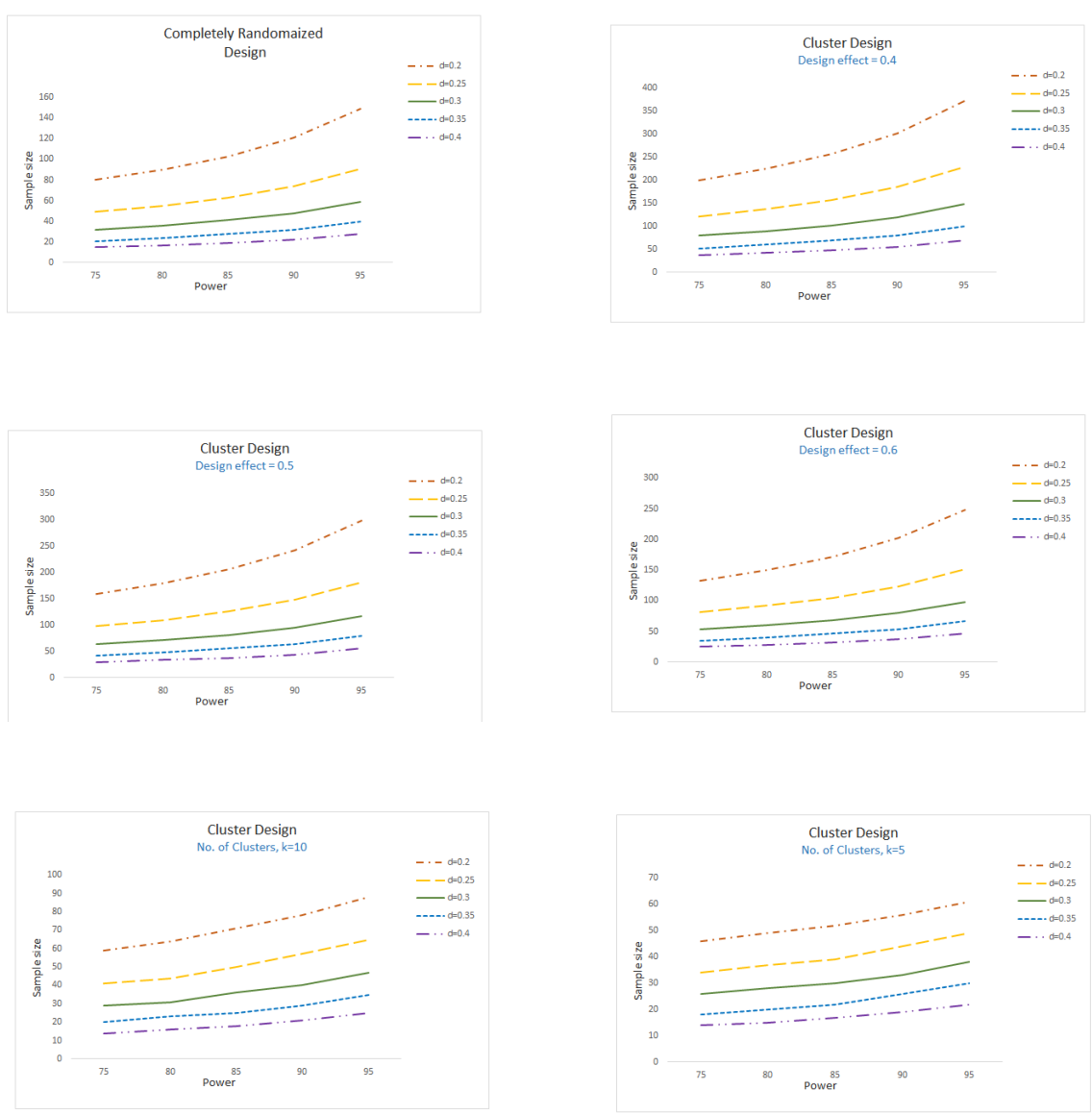

Figure 4. Sample sizes for different values of proportional difference and power

We can conclude that the sample size increases when the power of the test increases or the type I error decreases. Sample size also increases when the mean or proportional difference between the two study treatments decreases. The effective sample size is much higher when the design effect of the sampling method is smaller, particularly less than 1 . This means that 
the smaller the variance of the sampling technique, the larger the sample size will be. Also, for cluster sampling, sample size increases when the number of clusters increases.

\section{Data availability}

The data that support the findings of this study are available from the corresponding author upon reasonable request.

\section{REFERENCES}

A'hern R. (2001): Sample size tables for exact single-stage phase II designs. Statistics in Medicine 20(6): 859-866.

Ahnn S., Anderson S.J. (1998): Sample size determination in complex clinical trials comparing more than two groups for survival endpoints. Statistics in Medicine 17(21): 2525-2534.

Altman D.G. (1980): Statistics and ethics in medical research: III How large a sample? British Medical Journal, 281(6251): 1336.

Campbell M.K., Thomson S., Ramsay C.R., MacLennan G.S., Grimshaw, J.M. (2004): Sample size calculator for cluster randomized trials. Computers in Biology and Medicine 34(2): 113-125.

Carlin B.P., Berry S.M., Lee J.J., Muller P. (2010): Bayesian Adaptive Methods for Clinical Trials. CRC press.

Castellini G., Gianola S., Bonovas S., Moja L. (2016): Improving power and sample size calculation in rehabilitation trial reports: A methodological assessment. Archives of Physical Medicine and Rehabilitation 97(7): 1195-201.

Charles P., Giraudeau B., Dechartres A., Baron G., Ravaud P. (2009): Re-porting of sample size calculation in randomised controlled trials. British Medical Journal.

Copsey B., Thompson J. Y., Vadher K., Ali U., Dutton S. J., Fitzpatric R., Lamb S. E., Cook J. A, (2018): Sample size calculations are poorly conducted and reported in many randomized trials of hip and knee osteoarthritis: results of a systematic review. Journal of Clinical Epidemiology 104: 52-61.

Fleming T.R. (1982): One-sample multiple testing procedure for phase II clinical trials. Biometrics pages 143-151.

Jones S.R., Carley S., Harrison M. (2003): An introduction to power and sample size estimation. Emergency Medicine Journal.

Kadam P., Bhalerao S. (2010): Sample size calculation. International Journal of Ayurveda Research 1(1): 55-57.

Khan I., Sarker S., Hackshaw A. (2012): Smaller sample sizes for phase II trials based on exact tests with actual error rates by trading their nominal levels of significance and power. British Journal of Cancer 107(11): 1801-1809. 
Machin D., Campbell M., Fayers P., Pinol A. (1997a): Comparing paired groups for binary, ordered categorical and continuous outcomes. Sample Size Tables for Clinical Studies, 2nd edn. Oxford: Blackwell Science.

Machin D., Campbell M.J., Tan S.B., Tan S.H. (1997b): Comparing paired groups for binary, ordered categorical and continuous outcomes. Sample Size Tables for Clinical Studies, 3rd edn. pages 67-83.

Machin D., Campbell M.J., Tan S.-B., Tan S.-H. (2011): Sample Size Tables for Clinical Studies. John Wiley \& Sons.

Moussa M.A. (1988): Planning the size of survival time clinical trials with allowance for stratification. Statistics in Medicine, 7(5):559-569.

Sakpal, T. (2010). Sample size estimation in clinical trial. Perspectives in Clinical Research 1(2): 67-67.

Sargent D.J. Taylor J.M. (2009): Current issues in oncology drug development, with a focus on phase II trials. Journal of Biopharmaceutical Statistics 19(3): $556-562$.

Shahravan A., Haghdoost A., Rad M., Hashemipoor M., Shari M. (2014): Sample size calculation of clinical trials published in two leading endodontic journals. Iranian Endodontic Journal 9(1): 56-60.

Suresh K., Chandrashekara S. (2015): Sample size estimation and power analysis for clinical research studies. Journal of Human Reproductive Sciences 8(3): 186.

Wang S.-J., Hung H., O’Neill R.T. (2006): Adapting the sample size planning of a phase III trial based on phase II data. Pharmaceutical Statistics 5(2): 85-97.

Wittes J. (2002): Sample size calculations for randomized controlled trials. Epidemiologic Reviews 24(1): 39-53. 